\title{
Imaging Calreticulin for Early Detection of Immunogenic Cell Death During Anticancer Treatment
}

\author{
Dong-Yeon Kim*1, Ayoung Pyo*2, Misun Yun*3, Ramar Thangam*2, Sung-Hwan You², Ying Zhang², Ye-rim Jung ${ }^{2}$, \\ Dinh-Huy Nguyen ${ }^{2}$, Akhil Venu ${ }^{2}$, Hyeon Sik Kim ${ }^{4}$, Mee Sun Yoon ${ }^{5}$, Yeongjin Hong ${ }^{2,6}$, and Jung-Joon Min²,6 \\ ${ }^{I}$ College of Pharmacy and Research Institute of Pharmaceutical Science, Gyeongsang National University, Jinju, Korea; ${ }^{2}$ Institute for \\ Molecular Imaging and Theranostics, Department of Nuclear Medicine, Chonnam National University Medical School and Hwasun \\ Hospital, Hwasun, Korea; ${ }^{3}$ Microbiology and Functionality Research Group, Research and Development Division, World Institute of \\ Kimchi, Gwangju, Korea; ${ }^{4}$ Medical Photonics Research Center, Korea Photonics Technology Institute, Gwangju, Korea; ${ }^{5}$ Department \\ of Radiation Oncology, Chonnam National University Hwasun Hospital, Hwasun, Korea; and ${ }^{6}$ Department of Microbiology, \\ Chonnam National University Medical School, Hwasun, Korea
}

\begin{abstract}
Surface-exposed calreticulin (ecto-CRT) is a well-known "eat-me" signal exhibited by dying cells that contributes to their recognition and destruction by the immune system. We assessed the use of a CRT-specific binding peptide for imaging ecto-CRT during immunogenic cell death and its utility for early prediction of treatment response. Methods: A synthetic CRT-specific peptide, KLGFFKR (CRTpep), was labeled with fluorescein isothiocyanate or ${ }^{18} \mathrm{~F}$, and the characteristics of ecto-CRT were evaluated in a colon cancer cell line in vitro and in vivo. Results: In vitro flow cytometry, immunofluorescence staining, and in vivo small-animal PET imaging results showed that CRTpep detected preapoptotic cells treated with immunogenic drugs or radiation but not those treated with the nonimmunogenic drug or a nontherapeutic dose of immunogenic drug. Conclusion: The present results indicate that the CRT-specific peptide would enable the prediction of therapeutic response, thereby facilitating early decisions on continuation or discontinuation of immunogenic treatment.
\end{abstract}

Key Words: animal imaging; calreticulin; immunogenic cell death; molecular imaging; positron emission tomography; therapy response

J Nucl Med 2021; 62:956-960

DOI: 10.2967/jnumed.120.245290

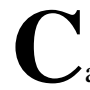

alreticulin (CRT) is a $\mathrm{Ca}^{2+}$-binding protein located mainly in the lumen of the endoplasmic reticulum (1). Surface exposure of CRT (ecto-CRT) can be induced by certain chemotherapeutic agents (e.g., oxaliplatin, mitoxantrone, and doxorubicin) and ionizing radiation and occurs within $1-4 \mathrm{~h}$ from the induction of immunogenic cell death, before the cells become apoptotic (2-7). By contrast, lethal stimuli that fail to elicit sufficient endoplasmic reticulum stress,

Received Mar. 17, 2020; revision accepted Oct. 22, 2020.

For correspondence or reprints contact either of the following: Jung-Joon Min, Chonnam National University Medical School and Hwasun Hospital, 264, Seoyang-ro, Hwasun-eup, Hwasun-gun, Jeollanam-do, 58128, Republic of Korea. E-mail: jjmin@jnu.ac.kr

Yeongjin Hong, Chonnam National University Medical School and Hwasun Hospital, 264, Seoyang-ro, Hwasun-eup, Hwasun-gun, Jeollanam-do, 58128, Republic of Korea.

E-mail: yjhong@chonnam.ac.kr

${ }^{*}$ Contributed equally to this work.

Published online Jan. 28, 2021.

COPYRIGHT (C) 2021 by the Society of Nuclear Medicine and Molecular Imaging. such as the nonimmunogenic drugs gemcitabine, mitomycin $\mathrm{C}$, and cisplatin, do not induce the immunogenic exposure of CRT on the cell surface unless an exogenous source of endoplasmic reticulum stress is provided $(8,9)$.

In cancer therapy, the ability to predict the response to treatment as early as possible is highly desirable. However, modern medical imaging such as CT and PET requires several weeks to months to predict responsiveness. Thus, molecular imaging technologies that can characterize and measure biologic processes at the cellular and molecular levels in living subjects $(10,11)$ are eagerly anticipated.

The KLGFFKR peptide motif in the cytoplasmic domain of an integrin that interacts with CRT to induce $\mathrm{Ca}^{2+}$ binding has potential as an imaging probe for targeting ecto-CRT (12). We labeled the KLGFFKR peptide sequence (CRT peptide [CRTpep]) with fluorescein isothiocyanate (FITC) or ${ }^{18} \mathrm{~F}$ and evaluated its ability to detect dying cancer cells in vitro and in vivo after treatment with chemotherapeutic drugs or radiation.

\section{MATERIALS AND METHODS}

\section{Radiochemistry for ${ }^{18} \mathrm{~F}$-CRTpep Synthesis}

CRT peptide (CRTpep), CRTpep-FITC (KLGFFKR and KLGFFKRFITC), and ${ }^{19} \mathrm{~F}$-CRTpep (reference compound) were synthesized by Anygen Co. (Supplemental Figs. 1-3; supplemental materials are available at http://jnm.snmjournals.org). $N$-succinimidyl 4-fluorobenzoate (SFB) and ethyl 4-(trimethyl-ammonium) benzoate $\left({ }^{18} \mathrm{~F}-\mathrm{SFB}\right.$ precursor) were prepared as previously described $(13,14)$, with some modifications. ${ }^{18} \mathrm{~F}$-CRTpep was synthesized using modifications of previously reported methods $(15,16)$. First, ${ }^{18} \mathrm{~F}-\mathrm{SFB}$ was synthesized to an approximately $30 \%-40 \%$ (non-decay-corrected) radiochemical yield; the radiochemical purity was more than $98 \%$. A fraction of the ${ }^{18} \mathrm{~F}-\mathrm{SFB}$ solution in high-performance liquid chromatography eluent was added to a reaction vessel and evaporated. CRTpep solution $(2.0 \mathrm{mg}, 2.235 \mu \mathrm{mol})$ in borate buffer $(\mathrm{pH} 8.5$, $0.1 \mathrm{M}$ ) was added to evaporated ${ }^{18} \mathrm{~F}-\mathrm{SFB}$ and incubated for $30 \mathrm{~min}$ at $40^{\circ} \mathrm{C}$. Finally, the crude ${ }^{18} \mathrm{~F}$-CRTpep $\left({ }^{18} \mathrm{~F}-\mathrm{SFB}\right.$-conjugated CRTpep) was cooled and injected into a semipreparative high-performance liquid chromatography system for purification (with the same gradient as used for the isolation of the reference compound). For identification of the ${ }^{18} \mathrm{~F}$ CRTpep, the collected high-performance liquid chromatography fraction (purified ${ }^{18} \mathrm{~F}-\mathrm{SFB}$ ) was coinjected with the ${ }^{19} \mathrm{~F}$-CRTpep. The radiochemical yield of ${ }^{18} \mathrm{~F}$-CRTpep was approximately $15 \%-20 \%$ 


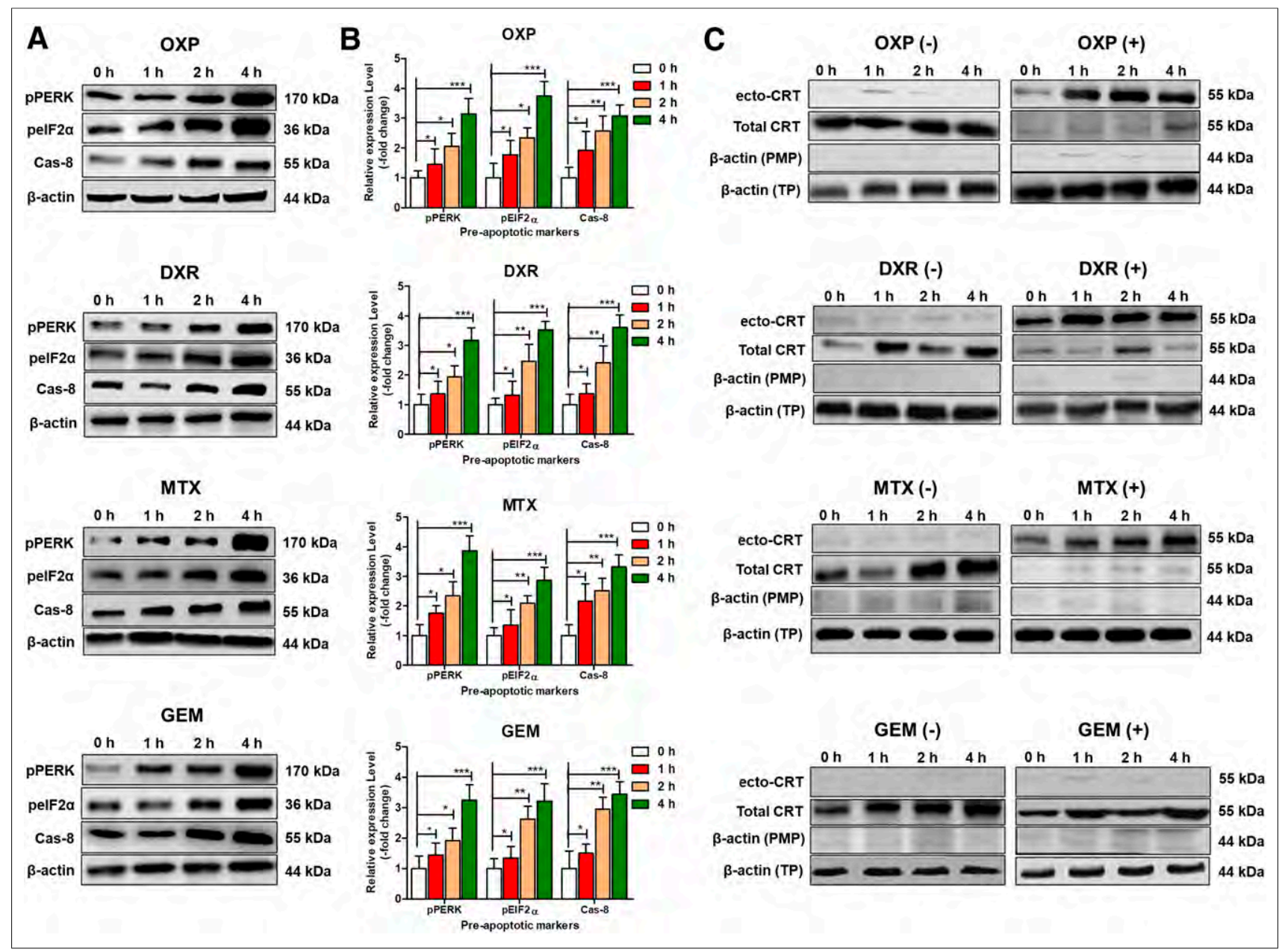

FIGURE 1. Expression of preapoptotic markers and CRT in CT26 cells after immunogenic treatment. CT26 cells were treated with oxaliplatin $(500 \mu \mathrm{M})$, doxorubicin $(25 \mu \mathrm{M})$, mitoxantrone $(3 \mu \mathrm{M})$, or gemcitabine $(15 \mu \mathrm{M})$ for $0,1,2$, and $4 \mathrm{~h}$. (A) Levels of preapoptotic proteins pPERK, pEIF2a, and Cas- 8 were analyzed by Western blotting. (B) Expression of preapoptotic-related markers pPERK, pEIF2a, and Cas-8 in CT26 cells was quantified using densitometry analysis of Western blots after treatment for $0,1,2$, and $4 \mathrm{~h}$ with oxaliplatin, doxorubicin, mitoxantrone, or gemcitabine. Relative expression was calculated to respective control using Student $t$ test, and level of expression was expressed as mean of 3 independent experiments. ${ }^{*} P<0.05$. ${ }^{\star \star} P<0.01$. ${ }^{\star \star \star} P<0.001$. (C) Western blot analysis of translocated CRT (ecto-CRT) in plasma membrane and total CRT in CT26 cell lysates with and without immunogenic treatment. CT26 cells were treated with or without immunogenic (oxaliplatin, doxorubicin, or mitoxantrone) and nonimmunogenic (gemcitabine) drugs for $0,1,2$, or $4 \mathrm{~h}$. DXR = doxorubicin; GEM = gemcitabine; MTX $=$ mitoxantrone; OXP = oxaliplatin; PMP = plasma membrane protein; TP = total protein.

(non-decay-corrected), and the radiochemical purity was more than $98 \%$ according to the analytic high-performance liquid chromatography system. ${ }^{18}$ F-CRTpep was dried, made isotonic with sodium chloride, and passed through a $0.2-\mu \mathrm{m}$ membrane.

\section{In Vivo Mouse Models and Small-Animal PET Studies}

The antitumor activity of the chemotherapy and radiation was evaluated in CT26 (murine colorectal carcinoma)-bearing BALB/c mice and B16F10 (murine melanoma)-bearing C57BL/6 mice when tumors reached approximately $100 \mathrm{~mm}^{3}$ after implantation. The mice were injected intravenously with doxorubicin $(5 \mathrm{mg} / \mathrm{kg}$ or $10 \mathrm{mg} / \mathrm{kg}$ ) and intraperitoneally with oxaliplatin $(5 \mathrm{mg} / \mathrm{kg})$ and gemcitabine $(15 \mathrm{mg} / \mathrm{kg}) 3$ times, with a 2 -d interval between each dose. Radiation therapy (15 Gy) was given once using a linear accelerator (CLINAC 21EX; Varian) emitting 10-MV x-rays to deliver a dose rate of $3 \mathrm{~Gy} / \mathrm{min}$ at a source-to-skin distance of $100 \mathrm{~mm}$ and a field size of $5 \times 5 \mathrm{~cm}^{-}$(17). Small-animal PET studies were performed immediately before and after treatment, $6 \mathrm{~d}$ after the start of ( $2 \mathrm{~d}$ after the last dose of ) oxaliplatin $(5 \mathrm{mg} / \mathrm{kg}, n=3)$, doxorubicin (5 or $10 \mathrm{mg} / \mathrm{kg}, n=3$, each), gemcitabine $(15 \mathrm{mg} / \mathrm{kg}$, $n=3$ ), or radiation therapy ( $15 \mathrm{~Gy}, n=3)$. Images were acquired 1 and $2 \mathrm{~h}$ after intravenous injection of ${ }^{18} \mathrm{~F}-\mathrm{FDG}(7.4 \mathrm{MBq} / 200 \mu \mathrm{L})$ and ${ }^{18} \mathrm{~F}$-CRTpep $(7.4 \mathrm{MBq} / 200 \mu \mathrm{L})$, respectively. Both studies were performed on the same animal at 8 -h intervals. All animal studies were conducted in accordance with the principles and procedures outlined in the National Institutes of Health Guide for the Care and Use of Laboratory Animals (18) and using protocols approved by the Animal Care and Use Committee of Chonnam National University.

\section{RESULTS}

\section{Preapoptotic Cell Death and Surface Exposure of CRT After Treatment}

Oxaliplatin at $500 \mu \mathrm{M}$, doxorubicin at $25 \mu \mathrm{M}$, mitoxantrone at $3 \mu \mathrm{M}$, and gemcitabine at $15 \mu \mathrm{M}$ were the doses chosen for analysis of preapoptotic markers and ecto-CRT based on apoptotic 


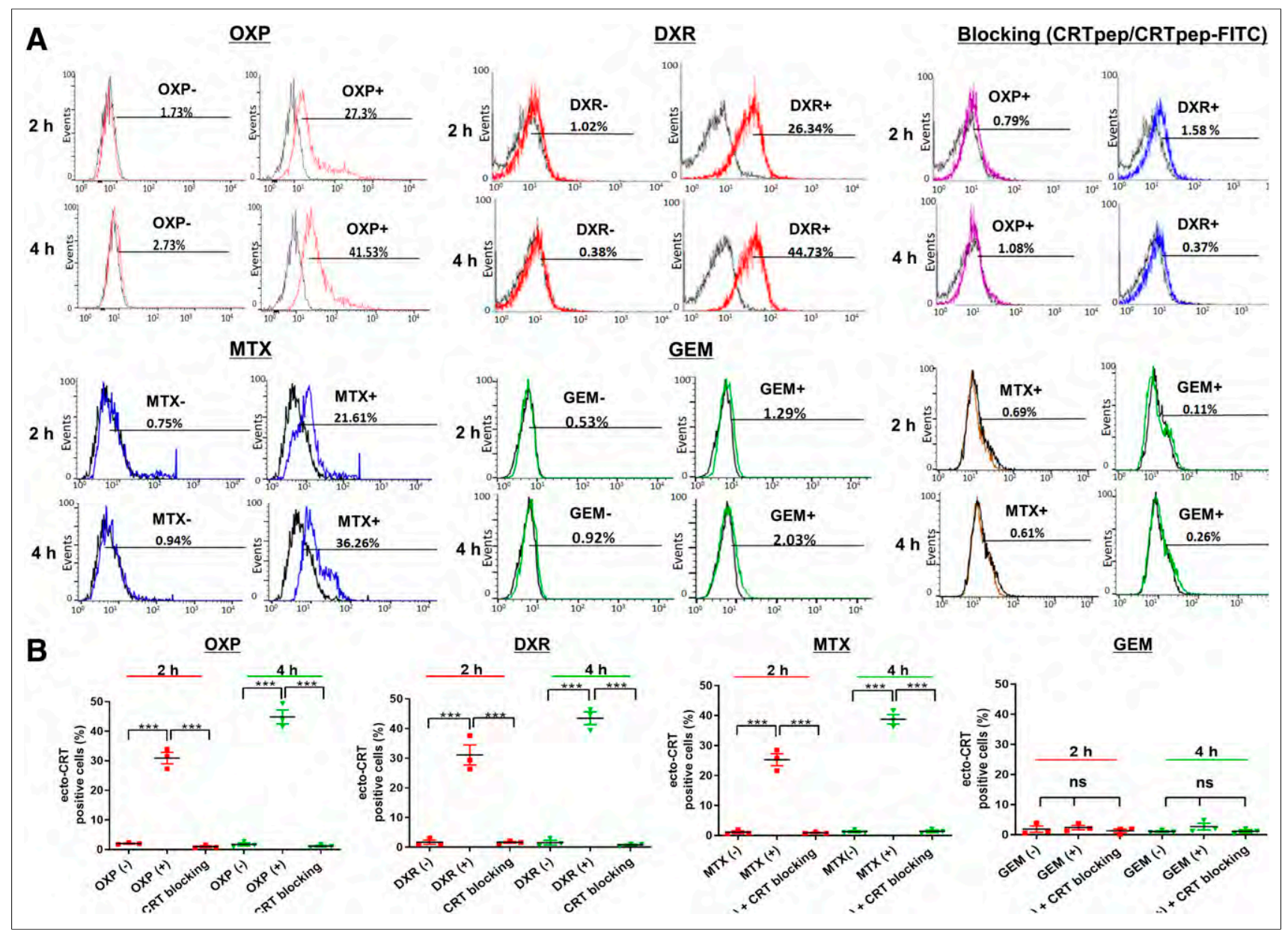

FIGURE 2. Flow cytometry analysis of CRTpep binding to ecto-CRT after immunogenic and nonimmunogenic drug treatment in CT26 cells. (A) Flow cytometry analysis of CRTpep binding to ecto-CRT after immunogenic and nonimmunogenic drug treatment in CT26 cells. Binding of FITCCRTpep to ecto-CRT in CT26 cells after 2 and $4 \mathrm{~h}$ of anticancer drug (immunogenic and nonimmunogenic) treatment was determined by flow cytometry. Percentage cellular uptake was based on detected mean fluorescence levels of untreated control cells. After anticancer drug treatment, CT26 cells were preincubated with CRTpep $(200 \mu \mathrm{M})$ for $1 \mathrm{~h}$, followed by incubation with FITC-CRTpep $(2 \mu \mathrm{M})$, and then were subjected to flow cytometry to detect uptake using fluorescence generated by ecto-CRT. (B) Quantitative assessment of binding of FITC-CRTpep to ecto-CRT in CT26 cells after 2 and $4 \mathrm{~h}$ of anticancer drug (immunogenic and nonimmunogenic) treatment that was determined by flow cytometry. Data are mean $\left( \pm\right.$ SD) fluorescence level. ${ }^{* \star} P<0.001(n=3)$. DXR = doxorubicin; GEM = gemcitabine; MTX = mitoxantrone; ns = not significant; OXP = oxaliplatin.

cell death rates of less than $20 \%$ at 2 and $4 \mathrm{~h}$ after drug exposure (Supplemental Figs. 4 and 5). The expression of endoplasmic reticulum stress and preapoptotic markers (pEIF2 $\alpha$, pPERK, and Cas-8) was significantly higher (2 and $4 \mathrm{~h}$ ) in oxaliplatin-, doxorubicin-, mitoxantrone-, and gemcitabine-treated cells than in untreated cells (Figs. 1A and 1B), demonstrating the early phase of apoptosis in response to the treatments with selected doses. Treatment with oxaliplatin, doxorubicin, mitoxantrone, and radiation (2, 5, and $10 \mathrm{~Gy})$ significantly increased ecto-CRT in CT26 cells, whereas the nonimmunogenic drug gemcitabine had no effect (Fig. 1C; Supplemental Fig. 6). In addition, doxorubicin treatment significantly increased the expression of ecto-CRT in CT26 xenografts (Supplemental Fig. 7). This finding demonstrated that CRT translocation was specifically induced by immunogenic drugs and radiation in preapoptotic CT26 cells.

\section{Specific Binding of CRTpep to Ecto-CRT}

The CRTpep was shown to have a micromolar affinity (dissociation constant, $1.868 \mu \mathrm{M}$; Supplemental Fig. 8). Specific binding of CRTpep to ecto-CRT was examined in CT26 and B16F10 cells before and treatment. Flow cytometry analysis and confocal fluorescence microscopy showed that CRTpep detected oxaliplatin-, doxorubicin-, and mitoxantrone-treated preapoptotic cells. By contrast, no significant binding was observed in gemcitabine-treated cells or in untreated cells (Figs. 2 and 3; Supplemental Figs. 9 and 10). Cell-surface binding of FITCCRTpep was decreased significantly by pretreatment with $200 \mu \mathrm{M}$ unlabeled CRTpep, indicating that the detected fluorescence corresponded to bound ecto-CRT (Figs. 2 and 3; Supplemental Figs. 9 and 10). Overall, these results indicate that CRTpep targets ectoCRT specifically in cells undergoing early immunogenic cell death.

\section{${ }^{18}$ F-CRTpep Small-Animal PET Imaging of Immunogenic Cancer Cell Death}

Subsequently, we used ${ }^{18} \mathrm{~F}$-CRTpep for in vitro and in vivo studies. ${ }^{18}$ F-CRTpep showed high stability for $2 \mathrm{~h}(>0 \%)$ 


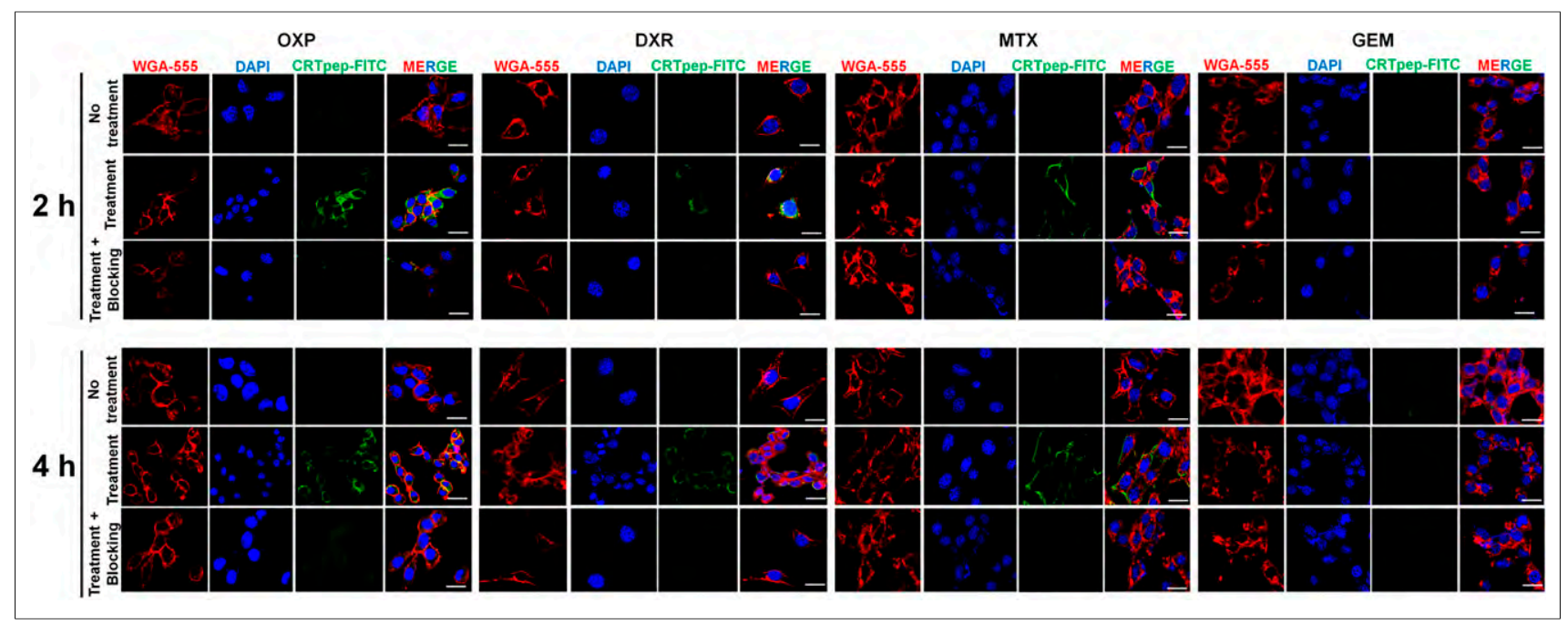

FIGURE 3. Immunofluorescence staining and analysis of CRTpep binding to ecto-CRT after immunogenic and nonimmunogenic drug treatment in CT26 cells. Binding of FITC-CRTpep to ecto-CRT in CT26 cells after 2 and $4 \mathrm{~h}$ of anticancer drug (immunogenic and nonimmunogenic) treatment was determined by confocal laser scanning microscopy ( $\times 40$ magnification) after immunofluorescence staining. Green $=$ FITCCRTpep; blue = 4',6-diamidino-2-phenylindole-stained nuclei; red $=$ cell membrane stained with wheat germ agglutinin 555 . (Scale bar $=$ $50 \mu \mathrm{m}$.) For blocking assay, anticancer drug-treated cells were further incubated with $200 \mu \mathrm{M}$ unlabeled CRTpep followed by $2 \mu \mathrm{M}$ FITCCRTpep. (Scale bar = $50 \mu \mathrm{m}$.) DAPI = 4',6-diamidino-2-phenylindole; DXR = doxorubicin; GEM = gemcitabine; $\mathrm{MTX}=$ mitoxantrone; OXP = oxaliplatin; WGA $=$ wheat germ agglutinin.

(Supplemental Fig. 11). Cellular uptake of ${ }^{18}$ F-CRTpep significantly increased after doxorubicin treatment (Supplemental Fig. 12). Next, we examined the potential of CRTpep for the detection of immunogenic cell death induction in vivo, with the ultimate aim of imaging tumor responses to immunogenic chemotherapy and radiotherapy. First, we assessed the tumor reductive effect of chemotherapeutic agents and radiotherapy. Doxorubicin, oxaliplatin, gemcitabine, or radiotherapy significantly suppressed tumor growth in CT26-bearing BALB/c mice (responder), whereas treatment with a nontherapeutic dose of doxorubicin $(5 \mathrm{mg} / \mathrm{kg})$ or phosphate-buffered saline (PBS) had no effect (nonresponder). Tumor volume between responder and nonresponder did not significantly differ until $6 \mathrm{~d}$ after treatment but became significantly different thereafter (Supplemental Fig. 13). Small-animal PET imaging showed a significant ${ }^{18}$ F-CRTpep signal in CT26 and B16F10 xenografts $6 \mathrm{~d}$ after treatment with a therapeutic dose of doxorubicin, oxaliplatin, or radiation (Figs. 4A and 4C; Supplemental Video 1; Supplemental Fig. 14). No significant signal was detected in the tumors of mice before treatment or in those treated with gemcitabine, a nontherapeutic dose of doxorubicin, or PBS. ${ }^{18}$ F-CRTpep uptake in tumors treated with a $10 \mathrm{mg} / \mathrm{kg}$ dose of doxorubicin was $29.7 \pm 2.8$-fold higher than that in the tumors of PBS-treated mice at posttreatment day 6 (Fig. 4C). PET imaging corresponded to the biodistribution study in doxorubicintreated CT26 xenograft mice (Supplemental Table 1). ${ }^{18} \mathrm{~F}-\mathrm{FDG}$ PET, which is widely used in the clinic, showed no significant difference in tumor uptake between the different treatments (Figs. 4B and 4D). Taken together, these results indicate that targeting ecto-CRT enables early measurement of treatment response in the context of immunogenic cell death.

\section{DISCUSSION}

A strong ecto-CRT signal was observed in responsive tumors as early as $6 \mathrm{~d}$ after the start of immunogenic treatment, whereas no significant signal was detected in unresponsive tumors or tumors treated with nonimmunogenic agents. Tumor uptake of ${ }^{18}$ F-CRTpep was observed before gross tumor suppression was observed and increased 30 -fold in doxorubicintreated tumors compared with untreated tumors. This uptake is considerably higher than that reported previously (19) for a molecular probe targeting surface-exposed histone ${ }^{1} \mathrm{H}$ (ApoPep-1), which showed a 1.6-fold increase in doxorubicin-treated tumors compared with untreated tumors. Accordingly, preapoptotic surface-exposed CRT would be more beneficial as a biomarker for determining the fate of cells after cancer treatment.

The present study, although lacking data related to the appraisal of an imaging agent (such as binding affinity measurement, pharmacokinetics, and biodistribution), provides proof of principle. Further studies using chemically modified CRTpep and engineered protein scaffolds with high binding affinity and in vivo stability are currently under way.

\section{CONCLUSION}

Imaging ecto-CRT may be an effective means of identifying tumor cells undergoing apoptosis in response to immunogenic therapy. The ability to recognize a preapoptotic state using PET at an early stage of immunogenic treatment has major implications for the early prediction of treatment response, which could improve the clinical outcomes of cancer therapy.

\section{DISCLOSURE}

This study was supported by grants from the National Research Foundation (NRF) of Korea (NRF-2017R1A2B3012157 and NRF2018M3A9H3024850) and the Pioneer Research Center Program (2015M3C1A3056410), funded by the Ministry of Science and ICT (MSIT). Dong-Yeon Kim was supported by the 


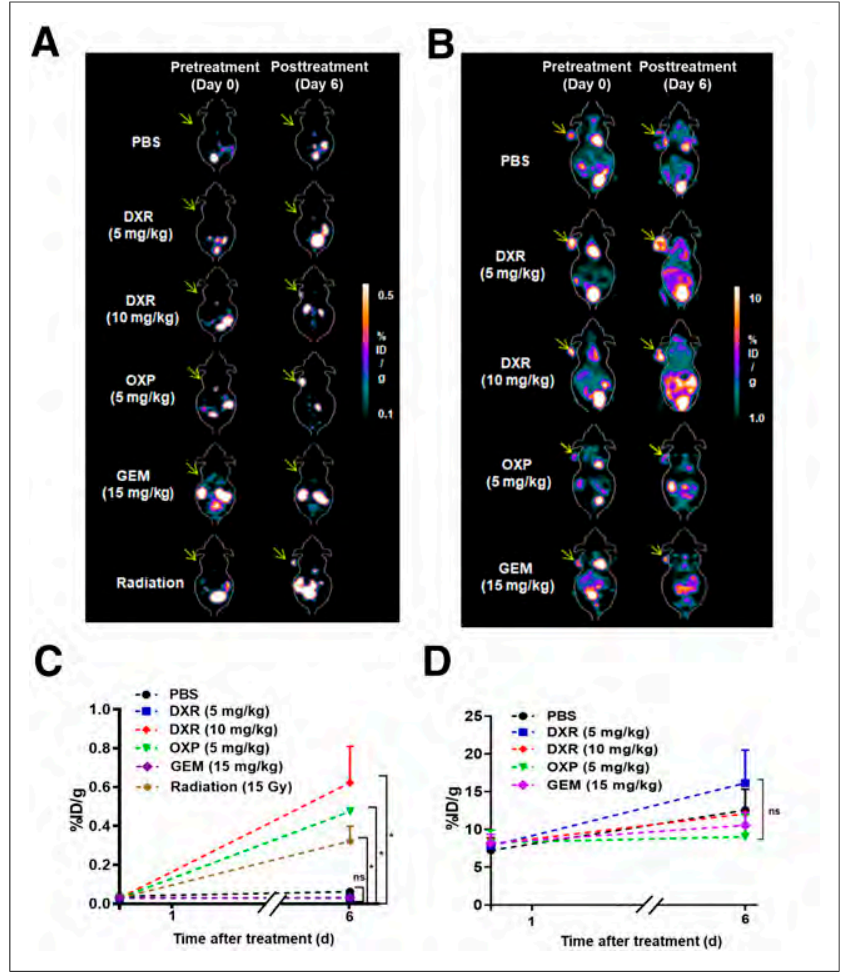

FIGURE 4. Assessment of immunogenic cell death by PET using ${ }^{18} \mathrm{~F}-$ CRTpep and ${ }^{18} \mathrm{~F}-\mathrm{FDG}$ in CT26 tumor-bearing mice. (A) Representative ${ }^{18} \mathrm{~F}$-CRTpep PET images of CT26-bearing mice. ${ }^{18} \mathrm{~F}$-CRTpep (7.4 MBq) was injected intravenously into mice before and at $6 \mathrm{~d}$ after chemotherapy or radiotherapy ( $n=4$ per group). Arrows indicate subcutaneous tumors. (B) Quantification of ${ }^{18} \mathrm{~F}-\mathrm{CRT}$ pep PET imaging signals in tumors before (day 0 ) and after treatment ( ${ }^{\star} P<0.05 ; P=0.4991$ and 0.9925 for PBS vs. doxorubicin [5 mg/kg] or gemcitabine on day 6 , respectively). (C) Representative ${ }^{18} \mathrm{~F}$-FDG PET images of CT26-bearing mice. ${ }^{18} \mathrm{~F}-\mathrm{FDG}$ was injected intravenously into same mice as used at $8 \mathrm{~h}$ after ${ }^{18} \mathrm{~F}$ CRTpep experiments ( $n=4$ per group). (D) Quantification of ${ }^{18} \mathrm{~F}-\mathrm{FDG}$ PET signals in tumors before (day 0 ) and after treatment. DXR $=$ doxorubicin; GEM = gemcitabine; ID = injected dose; MTX = mitoxantrone; $\mathrm{ns}=$ not significant; OXP $=$ oxaliplatin; $\mathrm{PBS}=$ phosphate-buffered saline.

NRF grant funded by MSIT (NRF-2020R1C1C1012379), and Ayoung Pyo was supported by the Basic Science Research Program through the NRF of Korea, funded by the Ministry of Education (NRF-2020R1I1A1A01070543). Yeongjin Hong was supported by an NRF of Korea grant, funded by the Korean government (MSIT; grant 2018R1A5A2024181). No other potential conflict of interest relevant to this article was reported.

\section{ACKNOWLEDGMENT}

We thank Hwa Youn Jang (Innovation Center for Molecular Probe Development in Chonnam National University Hwasun Hospital) for excellent research assistance
KEY POINTS

QUESTION: Does targeting ecto-CRT allow visualization of preapoptotic cells and eventually enable early measurement of treatment response in the context of immunogenic cell death?

PERTINENT FINDINGS: Imaging ecto-CRT was an effective means of identifying tumor cells undergoing apoptosis in response to immunogenic therapy. Strong tumor uptake of ${ }^{18} \mathrm{~F}-\mathrm{CRT}$ pep was observed before gross tumor suppression was observed, and uptake increased 30 -fold in doxorubicin-treated tumors compared with untreated tumors.

IMPLICATIONS FOR PATIENT CARE: The present results offer a new PET technology that would enable prediction of therapeutic response, thereby facilitating early decisions on continuation or discontinuation of immunogenic treatment

\section{REFERENCES}

1. Johnson S, Michalak M, Opas M, Eggleton P. The ins and outs of calreticulin: from the ER lumen to the extracellular space. Trends Cell Biol. 2001;11:122-129.

2. Garg AD, Nowis D, Golab J, Vandenabeele P, Krysko DV, Agostinis P. Immunogenic cell death, DAMPs and anticancer therapeutics: an emerging amalgamation. Biochim Biophys Acta. 2010;1805:53-71.

3. Panaretakis T, Kepp O, Brockmeier U, et al. Mechanisms of pre-apoptotic calreticulin exposure in immunogenic cell death. EMBO J. 2009;28:578-590.

4. Green DR, Ferguson T, Zitvogel L, Kroemer G. Immunogenic and tolerogenic cell death. Nat Rev Immunol. 2009;9:353-363.

5. Zitvogel L, Apetoh L, Ghiringhelli F, Kroemer G. Immunological aspects of cancer chemotherapy. Nat Rev Immunol. 2008;8:59-73.

6. Obeid M. ERP57 membrane translocation dictates the immunogenicity of tumor cell death by controlling the membrane translocation of calreticulin. J Immunol. 2008;181:2533-2543.

7. Obeid M, Tesniere A, Ghiringhelli F, et al. Calreticulin exposure dictates the immunogenicity of cancer cell death. Nat Med. 2007;13:54-61.

8. Garg AD, Krysko DV, Verfaillie T, et al. A novel pathway combining calreticulin exposure and ATP secretion in immunogenic cancer cell death. EMBO J. 2012;31:1062-1079.

9. Menger L, Vacchelli E, Adjemian S, et al. Cardiac glycosides exert anticancer effects by inducing immunogenic cell death. Sci Transl Med. 2012;4:143-199.

10. Brindle K. New approaches for imaging tumour responses to treatment. Nat Rev Cancer. 2008;8:94-107.

11. Willmann JK, Van Bruggen N, Dinkelborg LM, Gambhir SS. Molecular imaging in drug development. Nat Rev Drug Discov. 2008;7:591-607.

12. Kwon MS, Park CS, Choi K, et al. Calreticulin couples calcium release and calcium influx in integrin-mediated calcium signaling. Mol Biol Cell. 2000;11:1433-1443.

13. Dissoki S, Hagooly A, Elmachily S, Mishani E. Labeling approaches for the GE11 peptide, an epidermal growth factor receptor biomarker.J Labelled Comp Radiopharm. 2011;54:693-701.

14. Di Gialleonardo V, Signore A, Glaudemans AW, Dierckx RA, De Vries EF. $N$ (4-18 F-fluorobenzoyl)interleukin-2 for PET of human-activated T lymphocytes. $J$ Nucl Med. 2012;53:679-686.

15. Wang MW, Wang F, Zheng YJ, et al. An in vivo molecular imaging probe ${ }^{18} \mathrm{~F}-$ annexin B1 for apoptosis detection by PET/CT: preparation and preliminary evaluation. Apoptosis. 2013;18:238-247.

16. Tang G, Tang X, Wang X. A facile automated synthesis of $\mathrm{N}$-succinimidyl 4$\left[{ }^{18} \mathrm{~F}\right]$ fluorobenzoate $\left(\left[{ }^{18} \mathrm{~F}\right] \mathrm{SFB}\right)$ for ${ }^{18} \mathrm{~F}$-labeled cell-penetrating peptide as PET tracer. J Labelled Comp Radiopharm. 2010;53:543-547.

17. Jiang SN, Phan TX, Nam TK, et al. Inhibition of tumor growth and metastasis by a combination of Escherichia coli-mediated cytolytic therapy and radiotherapy. Mol Ther. 2010;18:635-642.

18. Guide for the Care and Use of Laboratory Animals. 8th ed. National Academy Press; 2011.

19. Wang K, Purushotham S, Lee JY, et al. In vivo imaging of tumor apoptosis using histone H1-targeting peptide. J Control Release. 2010;148:283-291. 